\title{
ON THE SEMISIMPLICITY OF GROUP ALGEBRAS, II
}

\author{
ORLANDO E. VILLAMAYOR
}

In a previous note [6] we found a partial solution to the problem of the semisimplicity of group algebras by proving that, if $K$ is a semisimple commutative algebra over the rationals and $G$ is a group which is locally finite over the center (i.e., if $C$ is the center of $G$, $G / C$ is locally finite), then the group algebra $K(G)$ is semisimple.

In this note we extend this result to a more general case, namely, when $G$ is a GSN-group (see definition in $\S 3$ ) which includes the case $G$ is an SN-group as defined in [5].

The main ideas and methods used in this note are, essentially, the same ones used ir [6]. The earlier theorem connecting homology and semisimplicity is now replaced by Lemma 2, which applies relative cohomology to be able to use the chain method given by Lemma 3 .

The connection theorem of $\$ 2$ and Lemma 3 are used to prove, furthermore, that the problem of the semisimplicity of the group algebras is reduced to the case of groups $G$ satisfying $G=[G, G]$.

1. Notations. Ring will mean associative ring with unit and a subring will always contain the unit of the big ring. In a ring, radical and semisimplicity are meant in the sense of [3], i.e., a ring will be called semisimple if its Jacobson radical is zero. If $A$ is a ring, the radical of $A$ will be noted by $\operatorname{Rad}(A)$.

In Relative Cohomology we shall follow [2]. In particular, if $R$ is a ring and $S$ a subring, $d(R, S)=0$ means that every $R$-module is $(R, S)$-projective, that is, every exact sequence of $R$-modules which splits as a sequence of $S$-modules, splits as a sequence of $R$-modules too.

2. The connecting theorem. Let $S$ be a normal subgroup in a group $G$, and suppose $\left\{g_{i}\right\}$ is a complete set of representatives of $G / S$ in $G$, then $\left\{g_{i}\right\}$ is a basis of $K(G)$ as a $K(S)$-module.

We shall say that the pair $(G, S)_{K}$ verifies the condition (A) (notation: $\left.(G, S)_{K} \in \mathbf{A}\right)$ if :

(A). For every $\alpha \in \operatorname{Rad} K(G)$, if $\alpha=\sum_{1}^{n} f_{i} g_{i}\left(f_{i} \in K(S)\right)$, then

$$
f_{i} \in \operatorname{Rad} K(S) \quad(1 \leqq i \leqq n) .
$$

Evidently, property (A) is independent of the choice of the representatives of $G / S$ in $G$; in fact, any change of representatives changes

Received by the editors April 9, 1958 and, in revised form, July 18, 1958. 
the elements $f_{i}$ by a factor in $S$, hence by an invertible element in $K(S)$.

Let $K(G)$ be the group algebra generated by any group $G$ over a commutative ring $K$. It is a well known result that, if $\alpha=\sum k_{i} g_{i}$ $\left(k_{i} \in K, g_{i} \in G\right)$ belongs to the radical of $K(G)$ and $S$ is any subgroup of $G$ containing the set $\left\{g_{i}\right\}$, then $\alpha$ is in the radical of $K(S)$. In fact, it is sufficient to see that the quasi-inverse of $\alpha$ is in $K(S)$. Let $\beta$ be the quasi-inverse of $\alpha$, i.e. $\alpha+\beta-\alpha \beta=0$. If $\left\{h_{j}\right\}$ is a fixed set of representatives of the left cosets of $G$ modulo $S$, then $\beta=\sum \gamma_{j} h_{j}$ $\left(\gamma_{j} \in K(S)\right.$ ), and $\alpha+\beta-\alpha \beta=0$ implies (if we call $\left.h_{0}=1\right) \alpha+\gamma_{0}-\alpha \gamma_{0}$ $=0$ and $\gamma_{j}-\alpha \gamma_{j}=0$. Since $\alpha$ is quasi-regular, $(1-\alpha) \gamma_{j}=0$ implies $\gamma_{j}=0(j \neq 0)$, and $\beta \in K(S)$.

This result leads to the following lemma:

Lemma 1. Let $G$ be a group and $S$ a normal subgroup of $G$. If for every $S^{\prime}$ such that $G \supseteq S^{\prime} \supseteq S$ and $S^{\prime} / S$ is finitely generated, all pairs $\left(S^{\prime}, S\right)_{K}$ verify property $(\mathbf{A})$, then $(G, S)_{K} \in \mathbf{A}$.

In fact, since every element of $K(G)$ is in some $K\left(S^{\prime}\right)$, the previous reasoning applies directly.

The following lemma is the main tool of this note. Its applications to group algebras will allow us to prove the theorems on semisimplicity of the next section.

Lemma 2. Let $R$ be a ring and $P$ a subring. Suppose $R$ is a free left$P$-module with a basis $X=\left\{u_{i}\right\}$ such that $u_{i} P=P u_{i}$ and the mapping $\sigma_{i}: p \rightarrow p_{i}^{\prime}$ given by $u_{i} p=p_{i}^{\prime} u_{i}$ is an automorphism of $P$. If $\sum_{1}^{n} u_{i} p_{i}$ $\in \operatorname{Rad} R$ and $d(R, P)=0$, then $p_{i} \in \operatorname{Rad} P(1 \leqq i \leqq n)$.

Proof. Let $V$ be any irreducible $P$-module and let

$$
W=R \otimes_{P} V .
$$

Then, $W$, as a $P$-module, is a direct sum $W=\sum \oplus u_{i} \otimes V$. Moreover, $u_{i} \otimes V$ is semilinearly isomorphic to $V$ (it may be obtained from $V$ by $\left.p \circ v=\sigma_{i}(p) \cdot v\right)$ and so $u_{i} \otimes V$ is irreducible too. Thus, $W$ is completely reducible as a $P$-module.

Let $U$ be any $R$-submodule of $W$, then the exact sequence

$$
0 \rightarrow U \rightarrow W \rightarrow W / U \rightarrow 0
$$

splits as a sequence of $P$-modules. Since $d(R, P)=0$, it also splits as a sequence of $R$-modules, i.e., every submodule has a complement in $W$, that is, $W$ is completely reducible as an $R$-module. Thus,

$\operatorname{Rad} R \cdot W=0$. 
Let $\sum u_{i} p_{i} \in \operatorname{Rad} R$, then $\sum u_{i} p_{i} \cdot W=0$. In particular, for every $m \in 1 \otimes V, \sum u_{i} p_{i} \cdot m=0$, that is, $\sum u_{i} p_{i}(1 \otimes v)=\sum u_{i} \otimes p_{i} v=0$, then $p_{i} v=0$ for every $v \in V$, hence $p_{i} \in \operatorname{Rad} P(1 \leqq i \leqq n)$.

This result has a direct application to group algebras in the following way:

Corollary 1. Let $1 \rightarrow S \rightarrow G \rightarrow H \rightarrow 1$ be an exact sequence of groups. If $H$ is finite of order $n$ and $K$ is uniquely divisible by $n$, then $(G, S)_{K}$ $\in \mathbf{A}$.

In fact, since $G / S$ is finite and $K$ is uniquely divisible by the order of $G / S$, Higman proved [2, Corollary p. 373] that $d(K(G), K(S))$ $=0 .{ }^{1}$ Since $S$ is normal in $G$ and any set of representatives of $G / S$ in $G$ verify the conditions imposed to the set $\left\{u_{i}\right\}$ in the lemma, the result follows.

By combining Lemma 1 and this corollary we obtain:

THEOREM 1. Let $1 \rightarrow S \rightarrow G \rightarrow H \rightarrow 1$ be an exact sequence of groups. If $H$ is localy finite and $K$ is uniquely divisible by the orders of the elements in $H$, then $(G, S)_{K} \in \mathbf{A}$.

In fact, Lemma 1 asserts that it is sufficient to prove condition $\mathbf{A}$ in $K\left(G^{\prime}\right)$ when $G^{\prime}$ runs over the inverse images of all finitely generated subgroups of $H$, we then have

$$
1 \rightarrow S \rightarrow G^{\prime} \rightarrow H^{\prime} \rightarrow 1
$$

with $H^{\prime}$ finite, and so the corollary asserts $\left(G^{\prime}, S\right)_{K} \in \mathbf{A}$.

3. The chain proofs. In a group $G$, complete systems of subgroups. jumps and normal systems are meant in the sense of $[5, \S 56$, p. 171].

Given a class $\mathbb{S}$ of groups, a normal system $\mathfrak{M}$ in $G$ will be called a (S-normal system if $S_{\alpha+1} / S_{\alpha} \in \mathbb{C}$ for every jump $\left(S_{\alpha+1}, S_{\alpha}\right)$ in the system.

The following lemma will be used to apply the results of the previous section to prove the semisimplicity of $K(G)$ when $G$ has a $\mathbb{E}$ normal system and $\mathfrak{E}$ is the set of all groups which are locally finite over their centers.

A group $G$ is called locally finite over its center $C$ if the quotient group $G / C$ is locally finite.

Lemma 3. Suppose a class (5) of groups is given, such that, for every exact sequence $1 \rightarrow S^{*} \rightarrow G^{*} \rightarrow H^{*} \rightarrow 1, H^{*} \in \mathcal{C}$ implies $\left(G^{*}, S^{*}\right)_{K} \in \mathbf{A}$ for

1 The proof given in [1, Theorem 12] may be used, with only minor alterations, to prove the converse of Higman's statement, namely, that $d(K(G), K(S))=0$ implies $G / S$ finite and $K$ uniquely divisible by the order of $G / S$. 
a given commutative ring $K$. If $G$ is a group, $S$ a normal subgroup in $G$ and $G / S$ has a (S-normal system, then $(G, S)_{K} \in \mathbf{A}$.

Proof. Let $\left\{g_{i}\right\}$ be a complete set of representatives of $G / S$ in $G$, $\sum_{1}^{n} f_{i} g_{i} \in \operatorname{Rad} K(G)\left(f_{i} \in K(S)\right)$ and $\bar{g}_{i}$ the image of $g_{i}$ in $G / S$.

Since the normal system in $G / S$ is complete, there is a jump $\left(\bar{S}_{\alpha}, \bar{S}_{\alpha+1}\right)$ such that $\bar{g}_{1}, \cdots, \bar{g}_{n} \in \bar{S}_{\alpha+1}$, at least one $\bar{g}_{i}$ is not in $\bar{S}_{\alpha}$ and $\bar{S}_{\alpha+1} / \bar{S}_{\alpha} \in \mathcal{C}$. (See [5, p. 171].) Suppose $\bar{g}_{1}, \cdots, \bar{g}_{k}$ is the subset of $\bar{g}_{1}, \cdots, \bar{g}_{n}$ contained in $\bar{S}_{\alpha}$ (thus, $\left.k<n\right)$. Let us call $S_{\alpha}$ the inverse image of $\bar{S}_{\alpha}$ in $G$.

Evidently, $\sum_{1}^{n} f_{i} g_{i} \in \operatorname{Rad} K\left(S_{\alpha+1}\right)$. Writing $\sum_{1}^{n} f_{i} g_{i}$ in terms of a $K\left(S_{\alpha}\right)$-basis of $K\left(S_{\alpha+1}\right)$, we obtain

$$
\sum_{1}^{n} f_{i} g_{i}=\sum_{1}^{k} f_{i} g_{i}+\text { terms not in } K\left(S_{\alpha}\right) .
$$

Since the sequence

$$
1 \rightarrow S_{\alpha} \rightarrow S_{\alpha+1} \rightarrow \bar{S}_{\alpha+1} / \bar{S}_{\alpha} \rightarrow 1
$$

is exact and $\bar{S}_{\alpha+1} / \bar{S}_{\alpha} \in \mathfrak{C}$, then $\sum_{1}^{\boldsymbol{k}} f_{i} g_{i} \operatorname{Rad} K\left(S_{\alpha}\right)$.

The proof of $f_{1} \in \operatorname{Rad} K(S)$ follows by induction on the length of $\sum f_{i} g_{i}$ (because $S_{\alpha} / S$ has also a (E-normal chain).

By multiplying $\sum_{1}^{n} f_{i} g_{i}$ by $g_{j}^{-1}$ and repeating the process we obtain $f_{j} \in \operatorname{Rad} K(S)(1 \leqq j \leqq n)$, that is, $(G, S)_{K} \in \mathbf{A}$.

Since the previous proof is, evidently, valid for descending chains, we can prove:

CoROllary 2. Let $1 \rightarrow S \rightarrow G \rightarrow H \rightarrow 1$ be an exact sequence of groups and $H$ free abelian. If $K$ is uniquely divisible by some integer $p$, then $(G, S)_{K} \in \mathbf{A}$.

In fact, if $H$ is free abelian, we have the descending chain

$$
\begin{aligned}
H & =Z \times Z \times Z \times \cdots, \\
H_{1} & =Z^{p} \times Z^{p} \times Z^{p} \times \cdots, \\
H_{2} & =Z^{p^{2}} \times Z^{p^{2}} \times Z^{p^{2}} \times \cdots \\
H_{3} & =Z^{p^{3}} \times Z^{p^{3}} \times Z^{p^{8}} \times \cdots
\end{aligned}
$$

with $\cap H_{i}=(1)$ and $H_{n} / H_{n+1}$ locally finite.

THEOREM 2. Let $1 \rightarrow S \rightarrow G \rightarrow H \rightarrow 1$ be an exact sequence of groups. If $K$ is a commutative algebra over the rationals and $H$ is locally finite over the center, then $(G, S)_{K} \in \mathbf{A}$. 
Proof. By Lemma 5 of [6], if $C$ is the center of $H, H$ has the following normal system:

$$
1 \subset M \subset C \subset H
$$

where $M$ is free abelian and both $C / M$ and $H / C$ are locally finite. Then, Theorem 1, Corollary 2 and Lemma 3 prove our statement.

According to the definition given by Kurosh [5, p. 182], a group $G$ is called an SN-group if it has a $\mathbb{E}$-normal system where $\mathbb{E}$ is the class of all commutative groups. We shall call GSN-group a group $G$ having a $\mathfrak{S}^{*}$-normal system where $\mathfrak{S}^{*}$ is the class of all groups which are locally finite over their centers.

By combining Lemma 3 and Theorem 2 we obtain

Theorem 3. If $K$ is a semisimple commutative algebra over the rationals and $G$ is a GSN-group, then the group algebra $K(G)$ is semisimple.

Let $D$ be the derived series of a group $G$ and $S$ the intersection of all subgroups of $G$ forming $\mathfrak{D}$, then $G / S$ is an $\mathrm{SN}$-group and $S=[S, S]$.

Then, Lemma 3 and Theorem 2 lead to

Theorem 4. Suppose $K$ is a commutative semisimple algebra over the rationals. If $K(S)$ is semisimple for every group $S$ such that $S=[S, S]$, then $K(G)$ is semisimple for every arbitrary group $G$.

REMARK 1. Lemma 3 and Theorem 2 give, also, an alternate proof of the semisimplicity of $K(G)$ when $G$ is a free group and $K$ is a semisimple algebra over the rationals. In fact, the derived series of $G$ has intersection $\{1\}\left[\mathbf{5}\right.$, p. 38]. Furthermore, the class $\left(^{N^{*}}\right.$ can be enlarged by adding all free groups.

REMARK 2. Theorem 2 gives a new proof of the result of [6], just by taking $S=1$.

\section{REFERENCES}

1. S. Eilenberg and T. Nakayama, On the dimension of modules and algebras, II, Nagoya Math. J. vol. 9 (1955) pp. 1-16.

2. D. G. Higman, Modules with a group of operators, Duke Math. J. vol. 21 (1954) pp. 369-376.

3. G. Hochschild, Relative homological algebra, Trans. Amer. Math. Soc. vol. 82 (1956) pp. 246-269.

4. N. Jacobson, Structure of rings, Amer. Math. Soc. Colloquium Publications, vol. 31, 1956.

5. A. G. Kurosh, The theory of groups, vol. II, Chelsea, 1956.

6. O. E. Villamayor, On the semisimplicity of group algebras, Proc. Amer. Math. Soc. vol. 9 (1958) pp. 621-627.

The Institute for Advanced Study And RUTGERS UNIVERSITY 